\title{
Walking on Gravel with Soft Soles using Linear Inverted Pendulum Tracking and Reaction Force Distribution
}

\author{
Adrien Pajon ${ }^{1}$, Stéphane Caron ${ }^{1}$, Giovanni De Magistris ${ }^{2}$, Sylvain Miossec ${ }^{3}$ and Abderrahmane Kheddar ${ }^{1,4}$
}

\begin{abstract}
Soft soles absorb impacts and cast ground unevenness during locomotion on rough terrains. However, they introduce passive degrees of freedom (deformations under the feet) that complexify the tasks of state estimation and overall robot stabilization. We address this problem by developing a control loop that stabilizes humanoid robots when walking with soft soles on flat and uneven terrain. Our closed-loop controller minimizes the errors on the center of mass (COM) and the zero moment point (ZMP) with an admittance control of the feet based on a simple deformation estimator. We demonstrate its effectiveness in real experiments on the HRP-4 humanoid.
\end{abstract}

\section{INTRODUCTION}

Walking with rigid limbs and feet forces contact transitions to be planned with nearly zero velocity to avoid shocks. This is a very conservative strategy that goes against dynamic motion. In order to absorb shocks at impacts and lower their propagation along the mechanical structure, humanoid robots integrate compliant mechanisms. A common solution is to add flexible mechanisms at the robot ankles [1], [2] that also protect the force sensor at each foot. However, such compliant mechanisms act like passive joints whose deformations [3] are not directly measurable. This makes the control of robot attitude difficult notably in complex maneuvers [4].

Rather than using compliant shock absorbers at the ankle, we investigate the use of thick soft soles under each foot, see early work in [5]. These soles absorb landing impacts and cast out ground unevenness, implying an increase of the contact surface. In order to generate a simulator of the deformable soles, we developed a deformation estimator [6] coupled with a corresponding Walking Pattern Generator (WPG) [7]. This simulator has been experimentally validated by successfully walking in open-loop with HRP-4 performing different experiments [8]. However, its timeconsuming computations prevented its application to online motion generation.

In this paper we develop a closed-loop controller for biped robots walking with soft soles. The goal of this controller is to minimize the tracking error in terms of COM velocity, COM and ZMP position by taking into account the deformation properties of materials under the feet. This results into an admittance control at the ankles whose gains are based on the sole stiffness in a nominal state from the deformation

\footnotetext{
${ }^{1}$ CNRS-UM2 LIRMM, IDH group, UMR5506, Montpellier, France.

2 IBM Research - Tokyo, IBM Japan

${ }^{3}$ Univ. Orléans, INSA-CVL, PRISME, EA 4229, F45072, France

4 CNRS-AIST Joint Robotics Laboratory (JRL), UMI3218/RL, Japan.

Corresponding author: adrien.pajon@gmail.com
}
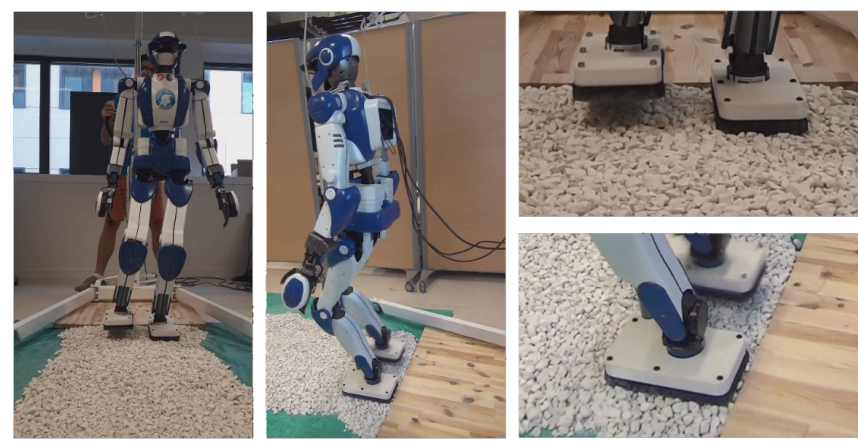

Fig. 1: Different views of HRP-4 walking on gravel with soft soles.

estimator. We tested our approach on a humanoid robot HRP4 walking on gravel, as depicted in Figure 1

\section{CONTROL FRAMEWORK STRUCTURE}

Our control pipeline is illustrated in Figure 2. Superscripts $d$ are used to denote desired references, $c$ control references and $i \in\{R, L\}$ right or left foot references. This pipeline goes as follows:

- A walking pattern generator (WPG) [7], [6], [8] outcomes desired COM $\boldsymbol{P}_{\text {сом }}^{d}$ and ZMP $\boldsymbol{P}_{\text {ZMP }}^{d}$ trajectories, along with the desired COM velocity $\dot{\boldsymbol{P}}_{\text {сом }}^{d}$ and the stiffness matrix of the soft sole $\boldsymbol{J}_{i}$.

- A ZMP-COM tracking controller (Section III) generates a control whole-body ZMP $\boldsymbol{P}_{\mathrm{ZMP}}^{c}$ that compensates both $\mathrm{COM}$ and ZMP errors between measurements and their respective WPG references.

- A ZMP-force distribution layer (Section IV) converts it into centers of pressure (CoP) under each foot in contact $\boldsymbol{P}_{\mathrm{CoP}_{i}}^{c}$, while the net reaction forces $\boldsymbol{F}^{c}$ is similarly distributed into contact forces $\boldsymbol{F}_{i}^{c}$.

- A reaction-force control layer (Section $\sqrt{\mathrm{V}}$ ) updates foot positions $\boldsymbol{P}_{i}^{d}$ and orientations $\boldsymbol{\Theta}_{i}^{d}$ to achieve the desired $\boldsymbol{P}_{\mathrm{CoP}_{i}}^{c}$ and $\boldsymbol{F}_{i}^{c}$ using admittance control [9].

Finally, a quadratic-programming (QP) whole-body controller finally produces joint motions that track the CoM and foot reference trajectories [10] from the force control layer and the COM trajectory.

\section{ZMP-COM CONTROL LAYER}

Our ZMP-COM control layer is based on [11], [12], [13]. We define a feedback controller on the state $\boldsymbol{x}=$ $\left[\begin{array}{lll}x_{\text {сом }} & \dot{x}_{\text {сом }} & x_{\mathrm{ZMP}}\end{array}\right]^{T}$ of COM position, COM velocity and ZMP 


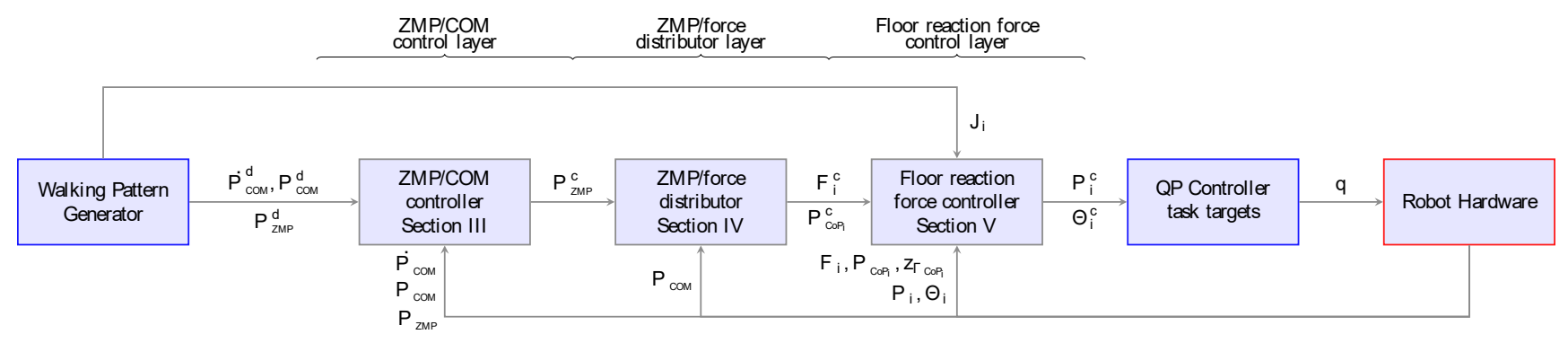

Fig. 2: Overview of the control loop. Superscripts $d$ and $c$ denote desired and control references, respectively, while robot measurements have none. $i \in\{R, L\}$ stands for right or left foot. $\boldsymbol{P}$ refers to positions, $\boldsymbol{F}$ to forces and $\boldsymbol{\Theta}$ to orientations.

position. We then proceed by pole placement in order to obtain the best COM-ZMP regulator [11], which is equivalent to a capture-point tracking controller [13].

\section{A. Linear inverted pendulum model}

The trajectories output by our WPG are based on the carttable model [14], that is:

$$
\boldsymbol{P}_{\mathrm{COM}}^{d}(t)=\left[\begin{array}{c}
x_{\mathrm{COM}}^{d}(t) \\
y_{\mathrm{COM}}^{d}(t) \\
z_{\mathrm{C}}
\end{array}\right], \boldsymbol{P}_{\mathrm{zMP}}^{d}(t)=\left[\begin{array}{c}
x_{\mathrm{ZMP}}^{d}(t) \\
y_{\mathrm{ZMP}}^{d}(t) \\
0
\end{array}\right]
$$

where $z_{\mathrm{C}}$ is the COM height above the ground. As the COM and ZMP are bound by a holonomic constraint to stay inside horizontal planes, all computations on the $x$-axis can be readily reproduced for the $y$-axis. In this model, the relationship between ZMP position and COM acceleration is given by:

$$
\ddot{x}_{\mathrm{COM}}(t)=\frac{g}{z_{\mathrm{C}}}\left(x_{\mathrm{COM}}(t)-x_{\mathrm{ZMP}}(t)\right)=\omega_{c}^{2}\left(x_{\mathrm{COM}}(t)-x_{\mathrm{ZMP}}(t)\right)
$$

with $g=9.81 \mathrm{~m} . \mathrm{s}^{-2}$ is the gravity constant.

To account for joint flexibilities and sole compliance, we assume that the real ZMP of the robot $\left(\boldsymbol{P}_{\mathrm{ZMP}}\right)$ lags behind the control ZMP $\left(\boldsymbol{P}_{\mathrm{ZMP}}^{c}\right)$. As in [12], [13], we model this delay as a low-pass filter whose transfer function is:

$$
\boldsymbol{P}_{\mathrm{ZMP}}(s)=\frac{1}{1+s T_{p}} \boldsymbol{P}_{\mathrm{ZMP}}^{c}(s)
$$

where $T_{p}$ is the low-pass time constant.

Let $\boldsymbol{x}=\left[\begin{array}{lll}x_{\text {Сом }} & \dot{x}_{\text {сом }} & x_{\mathrm{ZMP}}\end{array}\right]^{T}$ denote the state vector for the $x$-axis. Combining (3) and (2) yields the linear timeinvariant system:

$$
\dot{\boldsymbol{x}}(t)=\boldsymbol{A} \boldsymbol{x}(t)+\boldsymbol{B} x_{\mathrm{ZMP}}^{c}(t)
$$

with $\boldsymbol{A}=\left[\begin{array}{ccc}0 & 1 & 0 \\ \omega_{c}^{2} & 0 & -\omega_{c}^{2} \\ 0 & 0 & -1 / T_{p}\end{array}\right]$ and $\boldsymbol{B}=\left[\begin{array}{c}0 \\ 0 \\ 1 / T_{p}\end{array}\right]$

\section{B. Pole placement for ZMP-COM tracking control}

The following controller tracks the robot state $\boldsymbol{x}$ following desired state $\boldsymbol{x}^{d}$ given by the WPG. It eliminates the residual COM-state error:

$x_{\mathrm{ZMP}}^{c}(t)=\boldsymbol{K}_{\boldsymbol{l}} \boldsymbol{x}^{d}(t)-\boldsymbol{K}_{\boldsymbol{r}} \boldsymbol{x}(t)-k_{i} \int_{0}^{t}\left(x_{\mathrm{COM}}^{d}(t)-x_{\mathrm{COM}}(t)\right) \mathrm{d} t$ with $\boldsymbol{K}_{\boldsymbol{l}}=\left[\begin{array}{lll}k_{l 1} & k_{l 2} & k_{l 3}\end{array}\right], \boldsymbol{K}_{\boldsymbol{r}}=\left[\begin{array}{lll}k_{r 1} & k_{r 2} & k_{r 3}\end{array}\right]$ and $k_{i}$ the state feedback gains. Figure 3 represents the block diagram of the controller. The system enhanced by the integrator is:

$$
\left\{\begin{aligned}
\dot{\boldsymbol{x}}(t) & =\boldsymbol{A} \boldsymbol{x}(t)+\boldsymbol{B} x_{\mathrm{ZMP}}^{c}(t) \\
x_{\mathrm{COM}}(t) & =\boldsymbol{C} \boldsymbol{x}(t) \\
\dot{v}(t) & =x_{\mathrm{COM}}(t)-\boldsymbol{C} \boldsymbol{x}^{d}(t)
\end{aligned}\right.
$$

with $\boldsymbol{C}=\left[\begin{array}{lll}1 & 0 & 0\end{array}\right]$. Taking $\overline{\boldsymbol{x}}(t)=\left[\begin{array}{ll}\boldsymbol{x}(t) & v(t)\end{array}\right]^{T}$, Equation [5] with the controller writes:

$$
\left\{\begin{aligned}
\dot{\overline{\boldsymbol{x}}}(t) & =\overline{\boldsymbol{A}} \overline{\boldsymbol{x}}(t)+\overline{\boldsymbol{B}} x_{\mathrm{ZMP}}^{c}(t)+\overline{\boldsymbol{C}} \boldsymbol{x}^{d}(t) \\
x_{\mathrm{ZMP}}^{c}(t) & =-\overline{\boldsymbol{K}} \overline{\boldsymbol{x}}(t)+\boldsymbol{K}_{\boldsymbol{l}} \boldsymbol{x}^{d}(t)
\end{aligned}\right.
$$

with

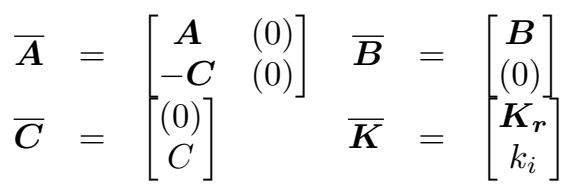

The extended Equation (6) can then be rewritten:

$$
\dot{\overline{\boldsymbol{x}}}(t)=(\overline{\boldsymbol{A}}-\overline{\boldsymbol{B} \boldsymbol{K}}) \overline{\boldsymbol{x}}(t)+\left(\overline{\boldsymbol{B}} \boldsymbol{K}_{\boldsymbol{l}}+\overline{\boldsymbol{C}}\right) \boldsymbol{x}^{d}(t)
$$

In static mode, we have:

$$
\begin{aligned}
& \lim _{t \rightarrow+\infty} \overline{\boldsymbol{x}}(t)=\overline{\boldsymbol{x}}^{\infty}=\left[\begin{array}{llll}
x_{\mathrm{COM}}^{\infty} & \dot{x}_{\mathrm{COM}}^{\infty} & x_{\mathrm{ZMP}}^{\infty} & z^{\infty}(=0)
\end{array}\right]^{T} \\
& \lim _{t \rightarrow+\infty} \boldsymbol{x}^{d}(t)=\boldsymbol{x}^{d}=\left[\begin{array}{lll}
x_{\mathrm{COM}}^{d} & \dot{x}_{\mathrm{COM}}^{d} & x_{\mathrm{zMP}}^{d}
\end{array}\right]^{T} \\
& 0=(\overline{\boldsymbol{A}}-\overline{\boldsymbol{B} \boldsymbol{K}}) \overline{\boldsymbol{x}}^{\infty}+\left(\overline{\boldsymbol{B}} \boldsymbol{K}_{\boldsymbol{l}}+\overline{\boldsymbol{C}}\right) \boldsymbol{x}^{d}
\end{aligned}
$$

This implies the gain relationships $k_{l 1}=k_{r 1}, k_{l 2}=k_{r 2}$ and $k_{l 3}=1+k_{r 3}$. Meanwhile, in a dynamic mode, we define the error as: $\overline{\boldsymbol{\epsilon}}(t)=\overline{\boldsymbol{x}}(t)-\overline{\boldsymbol{x}}^{\infty}$, so that:

$$
\dot{\overline{\boldsymbol{\epsilon}}}(t)=(\overline{\boldsymbol{A}}-\overline{\boldsymbol{B} \boldsymbol{K}}) \overline{\boldsymbol{\epsilon}}(t)
$$

In order to have a stable dynamic error that goes to zero in a limited amount of time, we need to choose the gains $\overline{\boldsymbol{K}}$ so that the matrix $(\overline{\boldsymbol{A}}-\overline{\boldsymbol{B K}})$ is stable. The choice of the gains $\overline{\boldsymbol{K}}$ are based on the eigenvalues $\left(\lambda_{1}, \lambda_{2}, \lambda_{3}, \lambda_{4}\right)$ of this matrix. From square matrices properties:

$$
\begin{aligned}
\operatorname{Tr}(\overline{\boldsymbol{A}}-\overline{\boldsymbol{B K}}) & =-\frac{1+k_{r 3}}{T_{p}}=\sum_{j=1}^{4} \lambda_{j} \\
\operatorname{det}(\overline{\boldsymbol{A}}-\overline{\boldsymbol{B K}}) & =-\frac{\omega_{c}^{2} k_{i}}{T_{p}}=\prod_{j=1}^{4} \lambda_{j}
\end{aligned}
$$


Next, the eigenvalues of a square matrix being the roots of its characteristic polynomial $\operatorname{det}(\overline{\boldsymbol{A}}-\overline{\boldsymbol{B} \boldsymbol{K}}-\lambda \boldsymbol{I})$, we have:

$$
\begin{aligned}
-\omega_{c}^{2}-\frac{k_{r 2} \omega_{c}^{2}}{T_{p}} & =\left(\lambda_{1} \lambda_{2}+\cdots+\lambda_{3} \lambda_{4}\right) \\
-\frac{\omega_{c}^{2}}{T_{p}}-\left(k_{r 1}+k_{r 3}\right) \frac{\omega_{c}^{2}}{T_{p}} & =\left(\lambda_{1} \lambda_{2} \lambda_{3}+\cdots+\lambda_{2} \lambda_{3} \lambda_{4}\right)
\end{aligned}
$$

The relation between eigenvalues and feedback gains is finally:

$$
\begin{aligned}
k_{r 1} & =-k_{r 3}-T_{p}\left(\frac{1}{T_{p}}-\frac{\lambda_{1} \lambda_{2} \lambda_{3}+\cdots+\lambda_{2} \lambda_{3} \lambda_{4}}{\omega_{c}^{2}}\right) \\
k_{r 2} & =-T_{p}\left(1+\frac{\lambda_{1} \lambda_{2}+\cdots+\lambda_{3} \lambda_{4}}{w_{c}^{2}}\right)^{2} \\
k_{r 3} & =-T_{p} \sum_{j=1}^{4} \lambda_{j}-1 \\
k_{i} & =-\frac{T_{p}}{\omega_{c}^{2}} \prod_{j=1}^{4} \lambda_{j}
\end{aligned}
$$

We conclude by noting that these eigenvalues are equal to the poles of the transfer function.

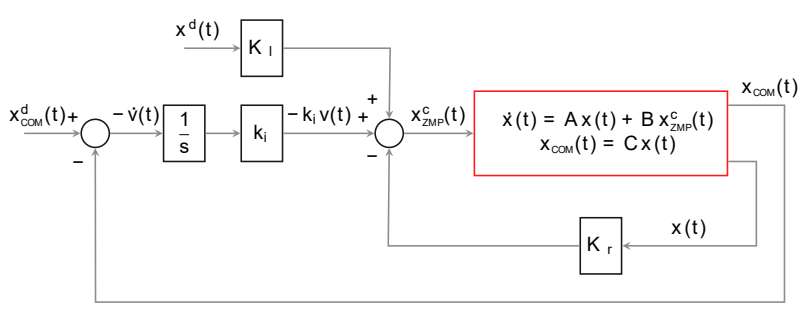

Fig. 3: ZMP-COM tracking controller: the state reference $\boldsymbol{x}^{d}$ of COM velocity, COM and ZMP position is compared to the measured state $\boldsymbol{x}$ in order to control the system (in red) with a ZMP control $x_{\mathrm{ZMP}}^{c}$

\section{ZMP-FORCE DISTRIBUTION LAYER}

The ZMP-COM controller issues control through a single whole-body ZMP, regardless of contact-stability constraints, that is to say, without enforcing the conditions thanks to which contacts neither slip nor tilt during locomotion. The goal of the ZMP-force distribution layer is two-fold: (1) distribute whole-body ZMP and resultant force at each contact, so as to (2) enforce contact-stability conditions on a percontact basis.

\section{A. Optimal force distribution and CoP placement}

From (2), the whole-body ZMP commands both the resultant moment and resultant force applied onto the robot. The latter is written as follows:

$$
\boldsymbol{F}=M\left(\ddot{\boldsymbol{P}}_{\mathrm{COM}}-\vec{g}\right)=M\left[\begin{array}{c}
\omega_{c}^{2}\left(x_{\mathrm{COM}}-x_{\mathrm{ZMP}}\right) \\
\omega_{c}^{2}\left(y_{\mathrm{COM}}-y_{\mathrm{ZMP}}\right) \\
g
\end{array}\right]
$$

where $M$ is the robot mass. We split this resultant force into forces $\boldsymbol{F}_{i}^{c}$ at each contact, and similarly the whole-body ZMP $\boldsymbol{P}_{\mathrm{ZMP}}^{c}$ into CoPs $\boldsymbol{P}_{\mathrm{ZMP}_{i}}$ at each contact. This ZMPforce distribution problem being underdetermined from an optimization standpoint, one can select solutions based on a desired cost function. Following [15], we define this function as the minimization of ankle torques. This choice together with simple approximations yields an analytical solution with a purely geometric construction:

$$
\left\{\begin{aligned}
F_{L}^{c z} & =M g \frac{\left\|\boldsymbol{P}_{R} \boldsymbol{P}_{\mathrm{ZMP}}^{o}\right\|}{\left\|\boldsymbol{P}_{R} \boldsymbol{P}_{L}\right\|} \\
\overrightarrow{\boldsymbol{N} \boldsymbol{P}_{\mathrm{ZMP}_{L}}} & =\frac{\left\|\boldsymbol{P}_{R} \boldsymbol{N}\right\|}{\boldsymbol{P}_{R} \boldsymbol{P}_{\mathrm{ZMP}}} \times \frac{\left\|\boldsymbol{P}_{R} \boldsymbol{P}_{\mathrm{ZMP}}^{o}\right\|}{\longrightarrow} \\
\overrightarrow{\boldsymbol{N} \boldsymbol{P}_{\mathrm{ZMP}_{R}}} & =\overrightarrow{\boldsymbol{P}_{L} \boldsymbol{P}_{\mathrm{ZMP}}} \times \frac{\left\|\boldsymbol{P}_{L} N\right\|}{\left\|\boldsymbol{P}_{R} \boldsymbol{P}_{\mathrm{ZMP}}^{o}\right\|} \\
F_{R}^{c z} & =M g-F_{L}^{c z}
\end{aligned}\right.
$$

where $\boldsymbol{P}_{\mathrm{ZMP}}^{o}$ is the orthogonal projection of $\boldsymbol{P}_{\mathrm{ZMP}}$ on the line $\left(\boldsymbol{P}_{R} \boldsymbol{P}_{L}\right), \boldsymbol{N}$ is the middle of the segment $\left[\boldsymbol{P}_{R} \boldsymbol{P}_{L}\right]$ and $F_{i}^{c z}$ is the vertical component of $\boldsymbol{F}_{i}^{c}$. Fig. 4 shows the graphical representation of these equations. We define $(L)$ and $(R)$ as the perpendiculars of $\left[\boldsymbol{P}_{L} \boldsymbol{P}_{R}\right]$ from $\boldsymbol{P}_{L}$ to $\boldsymbol{P}_{R}$.

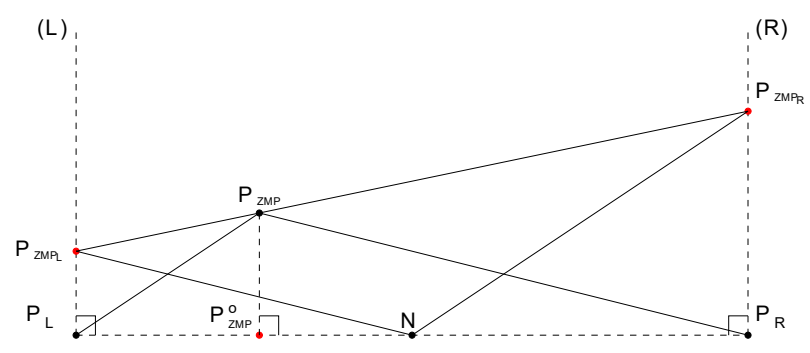

Fig. 4: Graphical description of the optimal $\boldsymbol{P}_{\mathrm{ZMP} L}$ and $\boldsymbol{P}_{\mathrm{ZMP} R}$ in DSP from 12.

\section{B. Satisfaction of ZMP constraints}

The non-tilting condition for foot contacts is characterized by the ZMP support area. This area is state-varying in general [16], notably when friction is limited. However, in our setting (cart-table model and larger friction over uneven ground thanks to sole compliance) we can safely approximate it by the convex hull of ground contact points [14].

When compensation terms in the COM-ZMP control law are too high, the whole-body control ZMP $\boldsymbol{P}_{\mathrm{zMP}}^{c}$ will lie outside this convex hull. In such cases, we project it back to the point $\boldsymbol{P}_{\mathrm{ZMP}}^{s}$ closest to it on the edge of the ZMP support area.

During single support phases (SSP), $\boldsymbol{P}_{\mathrm{ZMP}}^{c}$ is projected at the closest point on the foot shape in contact with the ground. Following our choice to minimize ankle torques during ZMPforce distribution, $\boldsymbol{P}_{\mathrm{ZMP}_{L}}$ and $\boldsymbol{P}_{\mathrm{ZMP}_{R}}$ during double support phases (DSP) are defined on the lines $(L)$ and $(R)$. They also must be inside their respective foot polygon. Those constraints delimit the existence of $\boldsymbol{P}_{\mathrm{ZMP}_{i}}$ into the segment [ $\left.\boldsymbol{P}_{i}^{1} \boldsymbol{P}_{i}^{2}\right]$ where $\boldsymbol{P}_{i}^{1}$ and $\boldsymbol{P}_{i}^{2}$ are the intersections of $(i)$ with the foot shape $i$. From the ZMP definition:

$$
\left\{\begin{aligned}
\left(x_{\mathrm{ZMP}_{R}}-x_{\mathrm{ZMP}}\right) F_{R}^{z} & + & \left(x_{\mathrm{ZMP}_{L}}-x_{\mathrm{ZMP}}\right) F_{L}^{z} & =0 \\
\left(y_{\mathrm{ZMP} R}-y_{\mathrm{ZMP}}\right) F_{R}^{z} & + & \left(y_{\mathrm{ZMP}_{L}}-y_{\mathrm{ZMP}}\right) F_{L}^{z} & =0 \\
F_{R}^{z} & + & F_{L}^{z} & =
\end{aligned}\right.
$$


which constraint the alignment of $\boldsymbol{P}_{\mathrm{ZMP}}, \boldsymbol{P}_{\mathrm{ZMP}_{L}}$ and $\boldsymbol{P}_{\mathrm{ZMP}_{R}}$. Hence, a reduced convex hull can be defined during DSP to constraint $\boldsymbol{P}_{\mathrm{ZMP}}^{c}$. The latter is defined by the convex polygon delimited by $\left(\boldsymbol{P}_{R}^{1} \boldsymbol{P}_{R}^{2} \boldsymbol{P}_{L}^{2} \boldsymbol{P}_{L}^{1}\right)$, as depicted in Figure 5 .

In the event where $\boldsymbol{P}_{\mathrm{zMP}}^{c}$ lies between $(L)$ and $(R)$, we choose to project it at the closest point on the reduced convex hull to keep the maximum dynamic on the COM. For $\boldsymbol{P}_{\mathrm{ZMP}}^{c}$ outside $(L)$ and $(R)$, we use the same projection as in a SSP case.

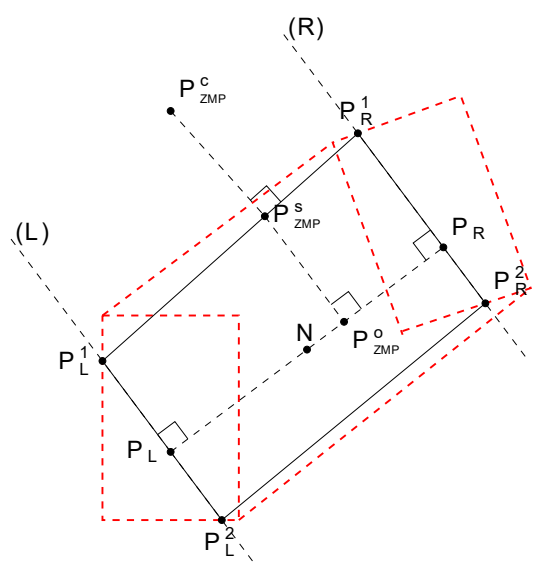

Fig. 5: Convex hull of ground contact points (dashed red) and reduced ZMP support area (black) in double support phases when ZMP-force distribution minimizes ankle torques. The control ZMP $\boldsymbol{P}_{\text {ZMP }}^{c}$ is projected to $\boldsymbol{P}_{\text {ZMP }}^{s}$.

\section{FLOOR REACTION FORCE CONTROL LAYER}

For humanoid robots that are controlled in position, force distribution control at the feet is realized by admittance control [9]. The output from an admittance controller consists of foot orientations and relative positions, whereas its intput is given by the resultant forces and CoPs computed by the ZMP-force distributor. Let us define:

$$
\delta_{i}=\left[\begin{array}{c}
\boldsymbol{P}_{i} \\
\boldsymbol{\Theta}_{i}
\end{array}\right], \mu_{i}=\left[\begin{array}{c}
\boldsymbol{F}_{i} \\
x_{\mathrm{CoP}_{i}} \\
y_{\mathrm{CoP}_{i}} \\
\Gamma_{\mathrm{CoP}_{i}}^{z}
\end{array}\right]
$$

Our model of the sole stiffness is given by:

$$
\dot{\mu}_{i}=\boldsymbol{J}_{i}\left(\mu_{i}\right) \dot{\delta}_{i}
$$

where the stiffness matrix $\boldsymbol{J}\left(\mu_{i}\right)$ is obtained by linearizaton of a finite element model (FEM) of the soft sole at $\mu_{i}$, as detailed in [8]. Equation (14) is a nonlinear model of the sole contact state that we approximate around a nominal $\mu_{i}^{0}$ state. However, we noticed in experiments that the rotational stiffness highly depends on the vertical force $F_{i}^{z}$. We then chose to model this dependance with the follwing model:

$$
\boldsymbol{J}_{i}\left(F_{i}^{z}\right)=\boldsymbol{J}_{i}\left(\mu_{i}^{0}\right)\left[\begin{array}{cc}
\boldsymbol{I} & (0) \\
(0) & k_{f}\left(F_{i}^{z}\right) \boldsymbol{I}
\end{array}\right]
$$

with $k_{f}\left(F_{i}^{z}\right)=F_{i}^{z} / F_{i}^{z_{0}}$ and $\boldsymbol{I}$ the identity matrix. Feedback control of such a system is equivalent to a damping:

$$
\dot{\delta}_{i}=\boldsymbol{D}_{i}^{-1}\left(\mu_{i}^{c}-\mu_{i}\right)
$$

We thus obtain the following closed-loop error transfer function:

$$
\Delta \dot{\mu}_{i}=-\boldsymbol{J}\left(F_{i}^{z}\right) \boldsymbol{D}_{i}^{-1} \Delta \mu_{i}
$$

We choose the poles as $\lambda_{a} \boldsymbol{I}$, so that $\boldsymbol{D}_{i}^{-1}=\lambda_{a} \boldsymbol{J}_{i}\left(F_{i}^{z}\right)^{-1}$. Figure 6 shows the block diagram of the resulting admittant controller.

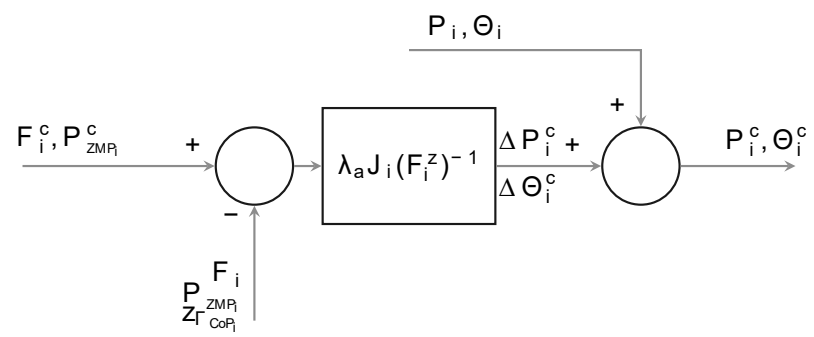

Fig. 6: Reaction force controller: the ZMP $\boldsymbol{P}_{\mathrm{ZMP}_{i}}^{c}$ and contact force $\boldsymbol{F}_{\boldsymbol{i}}^{\boldsymbol{c}}$ under the foot $i$ are compared to the robot state $\boldsymbol{P}_{\mathrm{zMP}}^{\boldsymbol{c}}$ and $\boldsymbol{F}_{\boldsymbol{i}}^{\boldsymbol{c}}$ in order to control in admittance the robot by generating foot $i$ relative position $\boldsymbol{P}_{\boldsymbol{i}}^{\boldsymbol{c}}$ and orientation $\boldsymbol{\Theta}_{\boldsymbol{i}}^{\boldsymbol{c}}$ control.

Note that, during SSP where the foot $i$ is not in contact with the ground, it is not controlled by the floor reaction force controller directly. Rather, $\boldsymbol{\Theta}_{i}$ is defined to put the foot $i$ horizontal and $\boldsymbol{P}_{i}$ is the interpolated trajectory of the ankle in the air to move the foot to the next planed foot-step.

\section{EXPERIMENTS}

\section{A. Experimental setup}

We perform walking experiments with the HRP-4 humanoid robot wearing custom feet with soft soles (Figure 7). These soles come as a replacement of the ankle shock absorbing system (hence the ankle leg link is rigid). The soles are made of a flexible foam. Combining the results of compression test and FEM simulation, we estimated their Young's modulus as $E=0.32 \mathrm{MPa}$ and Poisson ratio as $\nu=0.31$.

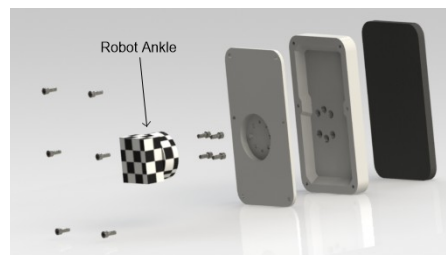

(a)

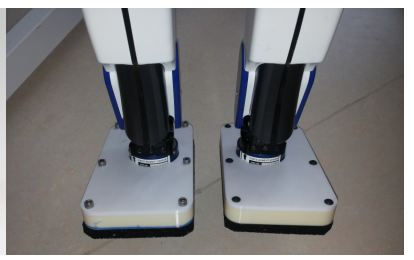

(b)
Fig. 7: (a): new HRP-4's feet; (b): photo of HRP-4's feet with soft soles

Experiments consist in a straightforward walk of 4 footsteps on a distance of $20 \mathrm{~cm}$ with an average velocity of $1.1 \mathrm{~cm} / \mathrm{s}$. An anti-windup is used to limit the error integration 
by defining a sliding integration windows of $400 \mathrm{~ms}$. The SSP and DSP durations given to the WPG are respectively set at $1 \mathrm{~s}$ and $2 \mathrm{~s}$. No ground information is given preliminary for walking, i.e. the whole experiment is terrain-blind.

The poles of the ZMP-COM tracking controller are set to $\left(-4,-4,-3,-\omega_{c}\right)$ while $T_{p}=0.11 \mathrm{~s}$. In order to minimize robot vibrations, $k_{f}$ is saturated between [0.5 - 1.5] during DSP and at 0.9 during SSP. HRP-4's low-level control frequency being $200 \mathrm{~Hz}$, we choose $\lambda_{a}=-200 \mathrm{~Hz}$. The nominal $\mu_{i}^{0}$ states are set to $\boldsymbol{F}_{i}^{0}=\left[\begin{array}{lll}0 & 0 & \frac{M g}{2}\end{array}\right]^{T}, \boldsymbol{P}_{\mathrm{CoP}_{i}}^{0}=\boldsymbol{P}_{i}$ and $\Gamma^{z 0}{ }_{\mathrm{CoP} i}=0$.

We used a simple COM state observer based on joint encoders, the assumption that contacts do not translate, and an estimate of the pelvis orientation frame provided by the robot software. This simple observer results in little mismatch between measures and actual COM location: in static equilibrium, projected COM and ZMP locations differ by roughly $2 \mathrm{~cm}$. Nonetheless, our closed-loop controller recovers from this static error and achieves walking with soft soles.

\section{B. Walking on flat floor and gravel}

Without a deformation estimator, HRP-4 was unable to walk on gravel without and with soft soles. With an offline deformation estimator, the robot manages to walk on flat ground but not on gravel. Furthermore, it then deals with a very limited range of perturbations/uncertainties.

With our closed-loop controller, the robot succeeded in walking on flat ground and on a bed of gravel with a granularity of $10 / 20 \mathrm{~mm}$. Figure 8 shows measurements for walking over gravel. These being similar to those observed over flat floor, to the exception of an increase in amplitude on ZMP-COM oscillations, the discussion will now focus on the earliest.

Figure $8 \mathrm{a}$ and $8 \mathrm{~b}$ represent the ZMP and COM in the direction of respectively $x$ - and $y$-axes controlled by the ZMP-COM control layer. The ZMP also remains within the support area. Some oscillations on the COM, and by extension the ZMP that controls it, are visible. They are mostly due to gravel irregularities and abrupt changes in contact surface during landing phases. The action of the ZMP control $\boldsymbol{P}_{\mathrm{ZMP}}^{c}$ is clearly visible in the controlled movement of ZMP in order to keep the COM close to its reference.

Figure $8 \mathrm{c}$ represents the vertical ground reaction forces (GRF) controlled by the admittance of the reaction force controller. The measure corresponds to the control with irregularities during transition between DSP and SSP when one foot takes off and lands on the ground. Some impacts are visible at landing and are quickly absorbed by the sole and the admittance controller. These observations validate the design of our deformation estimator model and floor reaction force controller.

Figures $8 \mathrm{~d}$, 8e and $8 \mathrm{f}$ represent the CoP under each foot, both controlled by the floor reaction control layer. Overall, measurements track well the references. As for the GRF, peaks of ZMP and CoP under the left foot appear due to an early landing foot. Oscillations in CoP and ZMP controls result from a combination of (1) gravel irregularities and (2) significant noise in COM velocity estimation. This noise is well distributed and with the mechanical lag, these vibrations are absorbed and have low impact on the actual measured ZMP.

The accompanying video shows experiments on the real robot: eight steps walking fully on gravel, as well as with a transition from wooden plank ground to gravel (Figure 1).

\section{CONCLUSION}

In this paper, we designed a closed-loop controller based on linear inverted pendulum tracking and an admittance control of ground reaction forces combined with a simple deformation estimator. We achieved walking with HRP-4 on flat and gravel grounds.

Robot stabilization depends on the identification of the mechanical lag time constant $T_{p}$ of ZMP response and the adaptation of the WPG to this time constant to choose the phase duration. Up to now, the WPG used for our experiments generates motion trajectories offline. We then plan to improve biped locomotion by using an adaptive online WPG (in terms of foot impact detection) coupled with an online estimation of the lags time constant and to change the phase durations in order to better stabilize the robot when the ground properties are changing. We will also test walking on other irregular terrains and larger steps.

In this paper, we added a thick squared soft sole under each foot of a humanoid robot. In the near future, we will test the developed controller on a robot with the optimized soles designed in [17].

\section{REFERENCES}

[1] K. Hirai, M. Hirose, Y. Haikawa, and T. Takenaka, "The development of honda humanoid robot," in IEEE International Conference on Robotics and Automation, vol. 2. Leuven, Belgium: IEEE, May 1998, pp. 1321-1326.

[2] K. Kaneko, F. Kanehiro, S. Kajita, H. Hirukawa, T. Kawasaki, M. Hirata, K. Akachi, and T. Isozumi, "Humanoid robot hrp-2," in IEEE International Conference on Robotics and Automation, New Orleans, USA, April 2004, pp. 1083-1090.

[3] O. Bruneau, F. B. Ouezdou, and J.-G. Fontaine, "Dynamic walk of a bipedal robot having flexible feet," in IEEE/RSJ International Conference on Intelligent Robots and Systems, vol. 1. Maui, USA: IEEE, 29 October 2001, pp. 512-517.

[4] J. Vaillant, A. Kheddar, H. Audren, F. Keith, S. Brossette, A. Escande, K. Bouyarmane, K. Kaneko, M. Morisawa, P. Gergondet, E. Yoshida, S. Kajita, and F. Kanehiro, "Multi-contact vertical ladder climbing with an hrp-2 humanoid," Autonomous Robots, vol. 40, no. 3, pp. 561-580, 2016.

[5] J. Yamaguchi and A. Takanishi, "Multisensor foot mechanism with shock absorbing material for dynamic biped walking adapting to unknown uneven surfaces," in IEEE International Conference on Multisensor Fusion and Integration for Intelligent systems, Washington DC, USA, December 1996, pp. 233-240.

[6] G. De Magistris, A. Pajon, S. Miossec, and A. Kheddar, "Humanoid walking with compliant soles using a deformation estimator," in International Conference on Robotics and Automation (ICRA), Stockholm, Sweden, 2016.

[7] A. Pajon, G. De Magistris, S. Miossec, K. Kaneko, and A. Kheddar, "A humanoid walking pattern generator for sole design optimization," in International Conference on Advanced Robotics (ICAR), Istanbul, Turkey, 2015. 


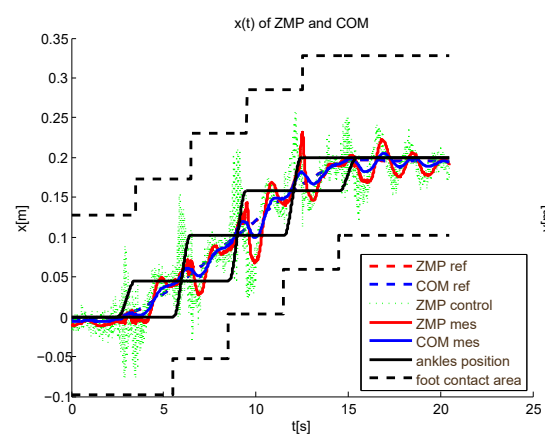

(a)

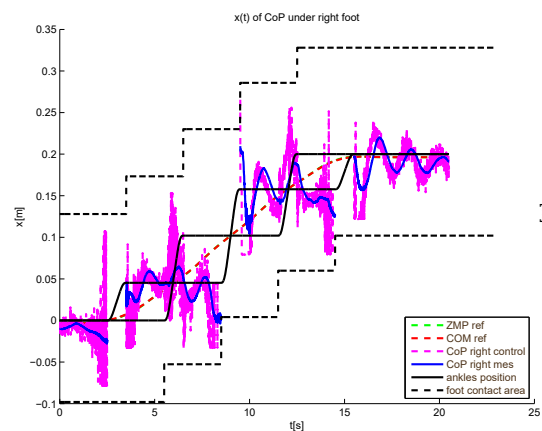

(d)

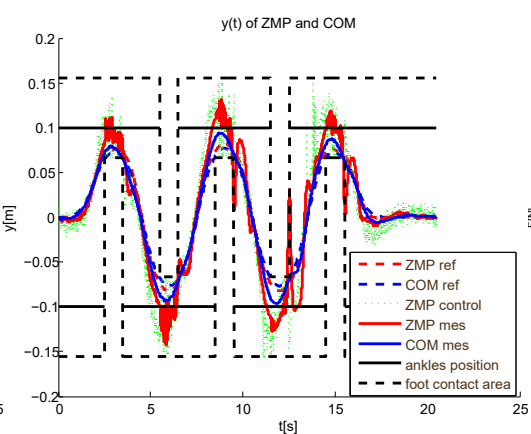

(b)

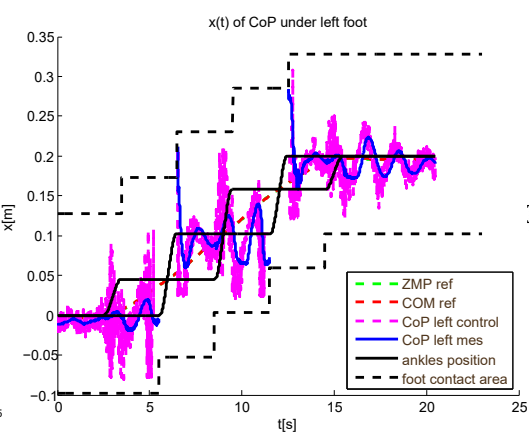

(e)

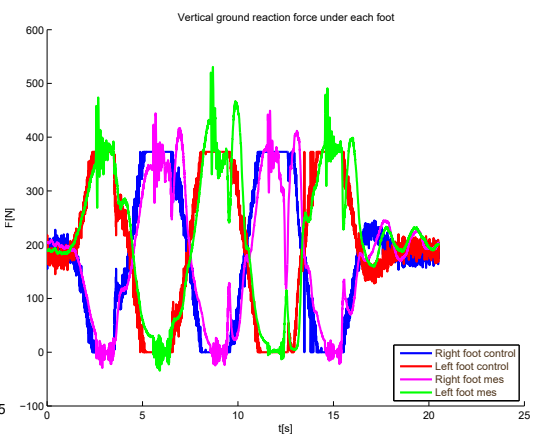

(c)

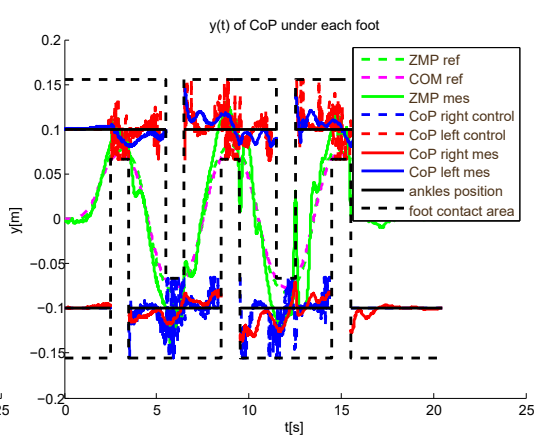

(f)

Fig. 8: Walking over gravel (granulometry: 10/20 mm): (a,b) ZMP and COM, (c) Vertical ground reaction forces and (d,e,f) CoP under each foot: measured compared with the references along $x / y$ axes during a four steps and $20 \mathrm{~cm}$ long.

[8] G. De Magistris, A. Pajon, S. Miossec, and A. Kheddar, "Optimized humanoid walking with soft soles," Robotics and Autonomous Systems, vol. 95 , pp. 52-63, 2017.

[9] J. De Schutter, H. Bruyninckx, W.-H. Zhu, and M. Spong, "Force control: a bird's eye view," Control Problems in Robotics and Automation, pp. 1-17, 1998.

[10] K. Bouyarmane, J. Vaillant, F. Keith, and A. Kheddar, "Exploring humanoid robots locomotion capabilities in virtual disaster response scenarios," in IEEE-RAS International Conference on Humanoid Robots, Osaka, 2012.

[11] T. Sugihara, "Standing stabilizability and stepping maneuver in planar bipedalism based on the best com-zmp regulator," in Robotics and Automation, 2009. ICRA'09. IEEE International Conference on. IEEE, 2009, pp. 1966-1971.

[12] S. Kajita, M. Morisawa, K. Miura, S. Nakaoka, K. Harada, K. Kaneko, F. Kanehiro, and K. Yokoi, "Biped walking stabilization based on linear inverted pendulum tracking," in IEEE/RSJ International Conference on Intelligent Robots and Systems, Taipei, Taiwan, October 18 - 22 2010, pp. 4489-4496.

[13] M. Morisawa, S. Kajita, F. Kanehiro, K. Kaneko, K. Miura, and K. Yokoi, "Balance control based on capture point error compensation for biped walking on uneven terrain," in Humanoid Robots (Humanoids), 2012 12th IEEE-RAS International Conference on. IEEE, 2012, pp. 734-740.

[14] S. Kajita and B. Espiau, Handbook of Robotics. Springer-Verlag, 2008, ch. Legged Robots, pp. 361-389.

[15] P. M. Wensing, G. Bin Hammam, B. Dariush, and D. E. Orin, "Optimizing foot centers of pressure through force distribution in a humanoid robot," International Journal of Humanoid Robotics, vol. 10, no. 03, p. 1350027, 2013.

[16] S. Caron, Q.-C. Pham, and Y. Nakamura, "Zmp support areas for multicontact mobility under frictional constraints," IEEE Transactions on Robotics, vol. 33, no. 1, pp. 67-80, 2017.

[17] G. De Magistris, S. Miossec, A. Escande, and A. Kheddar, "Design of optimized soft soles for humanoid robots," Robotics and Autonomous Systems, vol. 95, pp. 129 - 142, 2017. 